\title{
Local spectrum and local spectral radius of an operator at a fixed vector
}

\author{
by \\ JANKo BračIČ (Ljubljana) and Vladimír MÜller (Praha)
}

\begin{abstract}
Let $\mathcal{X}$ be a complex Banach space and $e \in \mathcal{X}$ a nonzero vector. Then the set of all operators $T \in \mathcal{L}(X)$ with $\sigma_{T}(e)=\sigma_{\delta}(T)$, respectively $r_{T}(e)=r(T)$, is residual. This is an analogy to the well known result for a fixed operator and variable vector. The results are then used to characterize linear mappings preserving the local spectrum (or local spectral radius) at a fixed vector $e$.
\end{abstract}

1. Introduction. Let $X$ be a complex Banach space and let $\mathcal{L}(X)$ be the Banach algebra of all bounded linear operators on $X$. For $T \in \mathcal{L}(X)$, let $\sigma(T)$, $\sigma_{\delta}(T)$, and $\sigma_{T}(x)$ be the spectrum, the surjectivity spectrum, and the local spectrum of $T$ at $x \in \mathcal{X}$, respectively. We denote by $r(T)=\lim _{k \rightarrow \infty}\left\|T^{k}\right\|^{1 / k}$ the spectral radius of $T$ and by $r_{T}(x)=\lim \sup _{k \rightarrow \infty}\left\|T^{k} x\right\|^{1 / k}$ the local spectral radius of $T$ at $x$. It is well known that $r(T)=\max \{|\lambda|: \lambda \in \sigma(T)\}$. On the other hand, $r_{T}(x) \geq \max \left\{|\lambda|: \lambda \in \sigma_{T}(x)\right\}$, and equality holds, for a given $T$ and every nonzero $x$, if $T$ has SVEP (see [6, Proposition 3.3.13]).

For $T \in \mathcal{L}(X)$ and $x \in \mathcal{X}$, it is easily seen that $\sigma_{T}(x) \subseteq \sigma_{\delta}(T) \subseteq \sigma(T)$. Moreover, the set of $x \in \mathcal{X}$ that satisfy $\sigma_{\delta}(T)=\sigma_{T}(x)$ and $r_{T}(e)=r(T)$ is residual (i.e., its complement is of the first category; see [8], and also [4], [6, Proposition 1.3.2]). In this paper, we are concerned with the "dual" question of how large the set $\mathcal{D}(e)=\left\{T \in \mathcal{L}(X): \sigma_{\delta}(T)=\sigma_{T}(e)\right\}$ is. It turns out (see Theorem 2.5) that $\mathcal{D}(e)$ is also residual whenever $e \neq 0$. Consequently, the set $\left\{T \in \mathcal{L}(X): r_{T}(e)=r(T)\right\}$, which contains $\mathcal{D}(e)$, is residual as well.

These results are later used to characterize linear mappings which preserve the local spectrum (local spectral radius) at a given vector $e \neq 0$. We show (Theorem 3.3) that a continuous linear surjection $\phi: \mathcal{L}(X) \rightarrow \mathcal{L}(X)$ satisfies $\sigma_{\phi(T)}(e)=\sigma_{T}(e)$, for every $T \in \mathcal{L}(\mathcal{X})$, if and only if there exists an invertible operator $A \in \mathcal{L}(X)$ such that $A e=e$ and $\phi(T)=A T A^{-1}$. This gives a solution to [5, Problem, p. 177].

2000 Mathematics Subject Classification: Primary 47A11; Secondary 47B49.

Key words and phrases: surjectivity spectrum, local spectrum, spectral radius, local spectral radius, linear maps preserving the local spectrum. 
Similarly, we show (Theorem 3.4) that a continuous linear surjection $\phi: \mathcal{L}(X) \rightarrow \mathcal{L}(X)$ satisfies $r_{\phi(T)}(e)=r_{T}(e)$, for every $T \in \mathcal{L}(X)$, if and only if there exist a complex number $c$ of modulus 1 and an invertible operator $A \in \mathcal{L}(X)$ such that $A e=e$ and $\phi(T)=c A T A^{-1}$. This result is an extension of [1, Theorem 1.1] to infinite-dimensional Banach spaces.

Throughout the paper we use standard notions and known facts from spectral and local spectral theory. The reader who is not familiar with these theories is referred to $[6,7]$.

2. Local/global spectrum and spectral radius. Let $X$ be a complex Banach space. It is well known that the mapping $T \mapsto \sigma(T)(T \in \mathcal{L}(\mathcal{X}))$ is upper semicontinuous. The same holds for the surjectivity spectrum $\sigma_{\delta}(T)=$ $\{z \in \mathbb{C}:(T-z) X \neq X\}$ as well. Namely, denote by $B x$ the unit ball in $X$ and let $k(T)=\sup \{r \geq 0: T B x \supseteq r B x\}$ be the surjectivity modulus of $T \in \mathcal{L}(X)$. By the Banach open mapping theorem, $T$ is surjective if and only if $k(T)>0$, which means that $\sigma_{\delta}(T)=\{z \in \mathbb{C}: k(T-z)=0\}$. Since $k: \mathcal{L}(X) \rightarrow[0, \infty)$ is a continuous function ([7, Proposition 9.9]) the set $\mathcal{S}=\{T \in \mathcal{L}(X): k(T)>0\}$ of all surjective operators is open. It follows, by [7, Proposition 6.9], that the surjectivity spectrum is an upper semicontinuous map.

Throughout this section, let $F \subseteq \mathbb{C}$ be a nonempty closed set and let $e \in \mathcal{X}$ be a nonzero vector. We denote by $A(F, X)$ the set of all bounded continuous functions $f: F \rightarrow X$ which are analytic on the interior of $F$. It is straightforward to see that $A(F, X)$, endowed with the norm $\|f\|_{\infty}=$ $\sup \{\|f(z)\|: z \in F\}$, is a Banach space. We shall consider $\mathcal{X}$ as the subset of all constant functions in $A(F, X)$; a vector $x \in \mathcal{X}$ is identified with the function $z \mapsto x(z \in F)$.

The open unit ball of $A(F, X)$ is denoted by $B_{A(F, X)}$.

Let

$$
\mathcal{S}(F)=\left\{T \in \mathcal{L}(X): \sigma_{\delta}(T) \cap F=\emptyset\right\}
$$

and let, for every $n \in \mathbb{N}$,

$$
\begin{aligned}
\mathcal{N}_{n}(F, e) & =\left\{T \in \mathcal{L}(X): e \notin n \overline{(T-z) B_{A(F, X)}},\right. \\
\mathcal{M}_{n}(F, e) & =\mathcal{S}(F) \cup \mathcal{N}_{n}(F, e) .
\end{aligned}
$$

We are going to show that $\mathcal{M}_{n}(F, e)$ is an open dense subset of $\mathcal{L}(X)$ for every $n \in \mathbb{N}$. In the proof we need the following observation.

Lemma 2.1. Let $S \in \mathcal{L}(X)$. If $\lambda \in \sigma_{\delta}(S)$, then, for every $\varepsilon>0$, there exists $T \in \mathcal{L}(X)$ such that $\|S-T\|<\varepsilon$ and $\overline{(T-\lambda) X} \neq X$.

Proof. There is no loss of generality to assume that $\lambda=0$. So, $0 \in \sigma_{\delta}(S)$ and consequently 0 is in the approximate point spectrum of $S^{*}$. Thus, there exists $\eta \in X^{*}$ such that $\|\eta\|=1$ and $\left\|S^{*} \eta\right\|<\varepsilon$. If $S^{*} \eta=0$, then $\overline{S X} \neq X$ 
and we can take $T=S$. Let $u \in X$ be such that $\|u\|<\varepsilon /\left\|S^{*} \eta\right\|$ and $\langle\eta, u\rangle=1$. We denote by $u \otimes \eta$ the rank-one operator $x \mapsto\langle\eta, x\rangle u$ on $X$. Note that its adjoint on $X^{*}$ is $\eta \otimes u: \xi \mapsto\langle\xi, u\rangle \eta$. Let $T=(I-u \otimes \eta) S$. Then $\|S-T\|=\left\|u \otimes\left(S^{*} \eta\right)\right\|<\varepsilon$ and $\langle\eta, T x\rangle=\left\langle S^{*}(I-\eta \otimes u) \eta, x\right\rangle=0$ for every $x \in \mathcal{X}$, which means $\overline{T X} \subseteq \operatorname{ker}(\eta) \neq X$.

Lemma 2.2. Let $S \in \mathcal{L}(\mathcal{X}), e \in \mathcal{X}, e \neq 0$. If $\lambda \in \sigma_{\delta}(S)$, then for every $\varepsilon>0$ there exists $T \in \mathcal{L}(X)$ such that $\|T-S\|<\varepsilon$ and $e \notin \overline{(T-\lambda) X}$.

Proof. By Lemma 2.1, there exists $T^{\prime} \in \mathcal{L}(X)$ such that $\left\|S-T^{\prime}\right\|<\varepsilon / 2$ and $\overline{\left(T^{\prime}-\lambda\right) X} \neq X$.

Let $\varepsilon^{\prime}=\min \left\{\varepsilon, 2\left\|T^{\prime}\right\|\right\}$. We shall show that there exists $T \in \mathcal{L}(\mathcal{X})$ satisfying $\left\|T^{\prime}-T\right\|<\varepsilon / 2$ and $e \notin \overline{(T-\lambda) X}$. This is obvious if $e \notin \overline{\left(T^{\prime}-\lambda\right) X}$. Assume therefore that $e \in \overline{\left(T^{\prime}-\lambda\right) X}$. Let $y$ be a closed subspace of $\overline{\left(T^{\prime}-\lambda\right) X}$ such that $\overline{\left(T^{\prime}-\lambda\right) X}=y \oplus \mathbb{C} e$. Fix $x \in \mathcal{X},\|x\|=1$, which is not in $\overline{\left(T^{\prime}-\lambda\right) \mathcal{X}}$. Let $\xi \in y^{\perp} \subseteq X^{*}$ be such that $\|\xi\|=1,\langle\xi, x\rangle \neq 0$, and $\langle\xi, e\rangle \neq 0$. Let $A=$ $I+\delta(x \otimes \xi)$, where $0<\delta<\varepsilon^{\prime} /\left(8\left\|T^{\prime}\right\|\right)$. Then $\|A-I\|=\delta<1$, which means that $A$ is invertible. The inverse is $A^{-1}=\sum_{k=0}^{\infty}(-\delta(y \otimes \xi))^{k}$ with the norm $\left\|A^{-1}\right\| \leq \sum_{k=0}^{\infty} \delta^{k}=1 /(1-\delta)$. Thus $\left\|A^{-1}-I\right\|=\left\|A^{-1}(I-A)\right\| \leq \delta /(1-\delta)$. Let $T=A T^{\prime} A^{-1}$. Then

$$
\left\|T-T^{\prime}\right\| \leq\left\|(A-I) T^{\prime} A^{-1}\right\|+\left\|T^{\prime}\left(A^{-1}-I\right)\right\|<\frac{2 \delta\left\|T^{\prime}\right\|}{1-\delta}<\frac{\varepsilon^{\prime}}{2} \leq \frac{\varepsilon}{2} .
$$

Moreover,

$$
\begin{aligned}
\overline{(T-\lambda) X} & =\overline{A\left(T^{\prime}-\lambda\right) A^{-1} X}=\overline{A\left(T^{\prime}-\lambda\right) X}=A y \vee\{A e\} \\
& =y \vee\{e+\delta\langle\xi, e\rangle x\},
\end{aligned}
$$

where $\vee$ denotes the closed linear span of two sets. If $e$ were in $\overline{(T-\lambda) X}$, then we would have $e=y+\alpha(e+\delta\langle\xi, e\rangle x)$ for some $y \in \boldsymbol{y}$ and $\alpha \in \mathbb{C}$. It would follow that $\alpha \delta\langle\xi, e\rangle x=(1-\alpha) e-y \in \overline{\left(T^{\prime}-\lambda\right) X}$ and consequently $\alpha=0$ because $x \notin \overline{\left(T^{\prime}-\lambda\right) X}$. However, we would then have a contradiction since $e$ cannot be equal to any $y \in \mathcal{y}$. Thus, $e \notin \overline{(T-\lambda) X}$.

Lemma 2.3. For every $n \in \mathbb{N}$, the set $\mathcal{M}_{n}(F, e)$ is an open and dense subset of $\mathcal{L}(X)$.

Proof. Since the surjectivity spectrum is an upper semicontinuous map it is obvious that $\mathcal{S}(F)$ is an open subset of $\mathcal{L}(X)$. Furthermore, if $T \in \mathcal{N}_{n}(F, e)$, then there exists $\varepsilon>0$ such that $\operatorname{dist}\left(e, n \overline{(T-z) B_{A(F, X)}}\right)>\varepsilon$. Let $T^{\prime} \in \mathcal{L}(\mathcal{X})$ be such that $\left\|T-T^{\prime}\right\|<\varepsilon /(2 n)$. Then, for every $f \in B_{A(F, X)}$,

$$
\left\|e-n\left(T^{\prime}-z\right) f\right\|_{\infty} \geq\|e-n(T-z) f\|_{\infty}-\left\|n\left(T^{\prime}-T\right)\right\|>\varepsilon-\varepsilon / 2=\varepsilon / 2,
$$

which means $T^{\prime} \in \mathcal{N}_{n}(F, e)$. Thus, $\mathcal{N}_{n}(F, e)$ is an open set and consequently $\mathcal{M}_{n}(F, e)$ is open as well. 
Now we shall show that $\mathcal{M}_{n}(F, e)$ is a dense subset of $\mathcal{L}(X)$. Let $S \in$ $\mathcal{L}(\mathcal{X})$ and $\varepsilon>0$ be arbitrary. We are looking for $T \in \mathcal{M}_{n}(F, e)$ satisfying $\|T-S\|<\varepsilon$. Of course, if $S \in \mathcal{M}_{n}(F, e)$, then there is nothing to prove. So, assume that $S \notin \mathcal{M}_{n}(F, e)$. It follows, in particular, that there exists $\lambda \in \sigma_{\delta}(S) \cap F$. By Lemma 2.2, there exists $T \in \mathcal{L}(X)$ such that $\|T-S\|<\varepsilon$ and $e \notin \overline{(T-\lambda) X} \neq X$.

It follows that $e \notin n \overline{(T-z) B_{A(F, X)}}$. Indeed, otherwise there would exist a sequence of functions $\left(f_{k}\right)_{k \in \mathbb{N}} \subset A(F, X)$ such that

$$
\lim _{k \rightarrow \infty}\left(\sup \left\{\left\|e-(T-z) f_{k}(z)\right\|: z \in F\right\}\right)=0 .
$$

In particular, we would have

$$
\lim _{k \rightarrow \infty}\left\|e-(T-\lambda) f_{k}(\lambda)\right\|=0,
$$

which is impossible since $e \notin \overline{(T-\lambda) X}$. We conclude that $T \in \mathcal{M}_{n}(F, e)$. It is obvious that $\|S-T\|<\varepsilon$.

Let

$$
\mathcal{N}(F, e)=\{T \in \mathcal{L}(X): e \notin(T-z) A(F, X)\}, \quad \mathcal{M}(F, e)=\mathcal{S}(F) \cup \mathcal{N}(F, e) .
$$

Lemma 2.4. For every nonempty closed set $F \subseteq \mathbb{C}$ and every nonzero vector $e \in X$, the set $\mathcal{M}(F, e)$ is a residual subset of $\mathcal{L}(X)$.

Proof. By the Baire category theorem, the set $\bigcap_{n=1}^{\infty} \mathcal{M}_{n}(F, e)$ is a dense $G_{\delta}$-set and hence residual.

Assume that $T \in \mathcal{L}(X)$ is not in $\mathcal{N}(F, e)$, which means that there exists $f \in A(F, X)$ such that $e=(T-z) f(z)$ for all $z \in F$. Let $n \in \mathbb{N}$ be such that $\|f\|_{\infty}<n$. Then $e \in n(T-z) B_{A(F, X)}$ and therefore $T \notin \mathcal{N}_{n}(F, e)$. Thus, $\bigcap_{n=1}^{\infty} \mathcal{N}_{n}(F, e) \subseteq \mathcal{N}(F, e)$ and consequently $\bigcap_{n=1}^{\infty} \mathcal{M}_{n}(F, e) \subseteq \mathcal{M}(F, e)$.

Theorem 2.5. If $e \in \mathcal{X}$ is a nonzero vector, then the set $\mathcal{D}(e)=\{T \in$ $\left.\mathcal{L}(X): \sigma_{\delta}(T)=\sigma_{T}(e)\right\}$ is residual.

Proof. Let $\left\{U_{k}: k \in \mathbb{N}\right\}$ be a countable base of open subsets of $\mathbb{C}$. For each $k \in \mathbb{N}$, the set $\mathcal{M}\left(\bar{U}_{k}, e\right)$ is a residual subset of $\mathcal{L}(X)$, by Lemma 2.4. Thus, $\bigcap_{k \in \mathbb{N}} \mathcal{M}\left(\bar{U}_{k}, e\right)$ is residual as well. Let $T \in \bigcap_{k \in \mathbb{N}} \mathcal{M}\left(\bar{U}_{k}, e\right)$. Towards a contradiction assume that $\lambda \in \sigma_{\delta}(T) \backslash \sigma_{T}(e)$. Hence $\lambda \in \rho_{T}(e)$ and therefore there exists an open neighbourhood $V$ of $\lambda$ and an analytic function $f$ : $V \rightarrow X$ such that $e=(T-z) f(z)$ for every $z \in V$. Let $k \in \mathbb{N}$ be such that $\lambda \in U_{k} \subset \bar{U}_{k} \subset V$. Denote by $g$ the restriction of $f$ to $\bar{U}_{k}$. Then $g \in A\left(\bar{U}_{k}, X\right)$ and $e=(T-z) g(z)$ for all $z \in \bar{U}_{k}$. It follows that $T \notin \mathcal{N}\left(\bar{U}_{k}, e\right)$. On the other hand, $T \notin \mathcal{S}\left(\bar{U}_{k}\right)$ since $\lambda \in \sigma_{\delta}(T) \cap \bar{U}_{k}$. Thus, $T \notin \mathcal{M}\left(\bar{U}_{k}, e\right)$, which contradicts the assumption.

Corollary 2.6. Let $e \in X$ be a nonzero vector. Then the set $\{T \in$ $\left.\mathcal{L}(X): r_{T}(e)=r(T)\right\}$ is residual. 


\section{Mappings preserving local spectrum and local spectral ra-} dius. Let $X$ be a Banach space. Linear mappings $\phi: \mathcal{L}(X) \rightarrow \mathcal{L}(X)$ preserving various properties have been extensively studied by a number of authors (see e.g. $[1,2,3,5]$ and references cited therein).

A linear mapping $\phi: \mathcal{L}(X) \rightarrow \mathcal{L}(X)$ is called a spectral isometry if $r(\phi(T))=r(T)$ for all $T \in \mathcal{L}(\mathcal{X})$. In [3, Theorem 1] the following result is proved.

TheOREM 3.1. Let $\phi: \mathcal{L}(X) \rightarrow \mathcal{L}(X)$ be a surjective spectral isometry. Then $\phi$ has one of the following two forms: either

(i) there are a complex number $c$ of modulus 1 and an invertible $A \in$ $\mathcal{L}(X)$ such that $\phi(T)=c A T A^{-1}$, for every $T \in \mathcal{L}(X)$, or

(ii) there are $c \in \mathbb{C},|c|=1$ and a bijection $A \in B\left(X^{*}, X\right)$ such that $\phi(T)=c A T^{*} A^{-1}$ for every $T \in \mathcal{L}(X)$ (in this case $X$ has to be reflexive).

If $\operatorname{dim} X<\infty$ and $e \in X$ is a fixed nonzero vector, then surjective linear mappings $\phi: \mathcal{L}(X) \rightarrow \mathcal{L}(X)$ satisfying $\sigma_{\phi(T)}(e)=\sigma_{T}(e)$ (respectively, $r_{\phi(T)}(e)=r_{T}(e)$ ), for all $T \in \mathcal{L}(\mathcal{X})$, were characterized in [5] (respectively, in [1]). We use the results from the previous section to generalize these results to the infinite-dimensional case.

Theorem 3.2. Let $X$ be a complex Banach space and $\phi: \mathcal{L}(X) \rightarrow \mathcal{L}(X)$ a continuous linear surjection. Let $e \in \mathcal{X}$ be a nonzero vector. Suppose that $\phi$ satisfies one of the following two conditions:

(i) $r_{\phi(T)}(e)=r_{T}(e)$ for all $T \in \mathcal{L}(X)$,

(ii) $\sigma_{\phi(T)}(e)=\sigma_{T}(e)$ for all $T \in \mathcal{L}(\mathcal{X})$.

Then $\phi$ is a spectral isometry.

Proof. First we prove that $r(\phi(T)) \geq r(T)$ for each $T \in \mathcal{L}(X)$, in both cases. Indeed, let $T \in \mathcal{L}(X)$ be arbitrary and choose $\lambda \in \sigma(T)$ such that $|\lambda|=r(T)$. Then $\lambda \in \sigma_{\delta}(T)$. By Lemma 2.2, for each $n \in \mathbb{N}$ there exists $T_{n} \in \mathcal{L}(X)$ such that $\left\|T_{n}-T\right\|<n^{-1}$ and $\lambda \in \sigma_{T_{n}}(e)$. By continuity of $\phi$ we have $\phi\left(T_{n}\right) \rightarrow \phi(T)$. In case (i), the upper semicontinuity of the spectral radius yields

$$
\begin{aligned}
r(\phi(T)) & \geq \limsup _{n \rightarrow \infty} r\left(\phi\left(T_{n}\right)\right) \geq \limsup _{n \rightarrow \infty} \max \left\{|\mu|: \mu \in \sigma_{\phi\left(T_{n}\right)}(e)\right\} \\
& =\limsup _{n \rightarrow \infty} \max \left\{|\mu|: \mu \in \sigma_{T_{n}}(e)\right\} \geq|\lambda|=r(T) .
\end{aligned}
$$

In case (ii), we have, similarly,

$$
\begin{aligned}
r(\phi(T)) & \geq \limsup _{n \rightarrow \infty} r\left(\phi\left(T_{n}\right)\right) \geq \limsup _{n \rightarrow \infty} r_{\phi\left(T_{n}\right)}(e)=\limsup _{n \rightarrow \infty} r_{T_{n}}(e) \\
& \geq|\lambda|=r(T) .
\end{aligned}
$$


Now we prove that $r(\phi(T))=r(T)$ for each $T \in \mathcal{L}(X)$. Let $T \in \mathcal{L}(\mathcal{X})$ and let $\lambda \in \sigma(\phi(T))$ satisfy $|\lambda|=r(\phi(T))$, which means $\lambda \in \sigma_{\delta}(\phi(T))$. By Lemma 2.2 , for each $n \in \mathbb{N}$ there exists $V_{n} \in \mathcal{L}(\mathcal{X})$ such that $\left\|V_{n}-\phi\left(T_{n}\right)\right\|<$ $n^{-1}$ and $\lambda \in \sigma_{V_{n}}(e)$. Since $\phi$ is continuous and surjective, by the Banach open mapping theorem there exists $k>0$ such that $\phi\left(B_{\mathcal{L}(X)}\right) \supset k \cdot B_{\mathcal{L}(X)}$ (here $B_{\mathcal{L}(X)}$ denotes the open unit ball in $\mathcal{L}(X)$ ). Therefore, for each $n$ there exists $T_{n} \in \mathcal{L}(\mathcal{X})$ such that $\left\|T_{n}-T\right\| \leq k^{-1}\left\|V_{n}-\phi(T)\right\| \leq k^{-1} n^{-1}$ and $\phi\left(T_{n}-T\right)=V_{n}-\phi(T)$. Thus $\left\|T_{n}-T\right\| \rightarrow 0$. In case (i), we have

$$
\begin{aligned}
r(T) & \geq \limsup _{n \rightarrow \infty} r\left(T_{n}\right) \geq \limsup _{n \rightarrow \infty} \max \left\{|\mu|: \mu \in \sigma_{T_{n}}(e)\right\} \\
& =\limsup _{n \rightarrow \infty} \max \left\{|\mu|: \mu \in \sigma_{\phi\left(T_{n}\right)}(e)\right\} \geq|\lambda|=r(\phi(T)),
\end{aligned}
$$

and in case (ii),

$$
\begin{aligned}
r(T) & \left.\left.\geq \limsup _{n \rightarrow \infty} r\left(T_{n}\right) \geq \limsup _{n \rightarrow \infty} r_{T_{n}}(e)\right\}=\limsup _{n \rightarrow \infty} r_{\phi\left(T_{n}\right)}(e)\right\} \\
& \geq|\lambda|=r(\phi(T)) .
\end{aligned}
$$

Hence $r(\phi(T))=r(T)$ for each $T \in \mathcal{L}(X)$.

Theorem 3.3. Let $e \in X$ be a nonzero vector. Let $\phi: \mathcal{L}(X) \rightarrow \mathcal{L}(X)$ be a surjective continuous linear mapping. Then $\sigma_{\phi(T)}(e)=\sigma_{T}(e)$ for all $T \in \mathcal{L}(X)$ if and only if there exists an invertible operator $A \in \mathcal{L}(X)$ such that $A e=e$ and $\phi(T)=A T A^{-1}$ for all $T \in \mathcal{L}(X)$.

Proof. If $\phi(T)=A T A^{-1}$ for every $T \in \mathcal{L}(X)$, where $A \in \mathcal{L}(X)$ is an invertible operator such that $A e=e$, then $\sigma_{T}(e)=\sigma_{\phi(T)}(e)$ for every $T \in$ $\mathcal{L}(X)$. Indeed, let $U \subseteq \rho_{T}(e)$ be an open set for which there exists an analytic function $f: U \rightarrow X$ that satisfies $(z-T) f(z)=e$ for all $z \in U$. Then $\tilde{f}(z)=$ $A f(z)$ is an analytic function on $U$ as well and it satisfies $\left(z-A T A^{-1}\right) \tilde{f}(z)=$ $e(z \in U)$. Thus, $U \subseteq \rho_{\phi(T)}(e)$. This proves the inclusion $\rho_{T}(e) \subseteq \rho_{\phi(T)}(e)$. The inclusion $\rho_{T}(e) \supseteq \rho_{\phi(T)}(e)$ is proved similarly.

Now we shall prove the opposite implication. Let $\phi: \mathcal{L}(X) \rightarrow \mathcal{L}(X)$ be a continuous linear surjection preserving the local spectrum at $e$. By the previous theorem, $\phi$ is a spectral isometry and, by Theorem 3.1, $\phi$ has one of the two forms mentioned there. Moreover, since $\phi(I)=c I$, where $I$ is the identity operator on $\mathcal{X}$, one has $\{1\}=\sigma_{I}(e)=\sigma_{\phi(I)}(e)=\sigma_{c I}(e)=\{c\}$ and consequently $c=1$. By [5, Lemma 5], case (ii) from Theorem 3.1 is not possible. Thus, $\phi(T)=A T A^{-1}$, where $A \in \mathcal{L}(\mathcal{X})$ is an invertible operator. Note that $A$ is not uniquely determined, we can replace it by $\lambda A$, where $\lambda$ is an arbitrary nonzero number. Thus, we have to see that $\lambda A e=e$ for some nonzero $\lambda$. Let $\xi \in X^{*}$ be such that $\langle\xi, e\rangle=1$ and let $T=e \otimes \xi$. Then $\sigma_{\phi(T)}(e)=\sigma_{T}(e)=\{1\}$. Since $T$ has SVEP there exists an analytic function $f: \mathbb{C} \backslash\{1\} \rightarrow X$ that satisfies $(z-\phi(T)) f(z)=e(z \in \mathbb{C} \backslash\{1\})$. It is easy to see that $\lambda A e=e$, for some $\lambda \neq 0$, if $z=0$ in the last equality. 
Theorem 3.4. Let $e \in X$ be a nonzero vector. Let $\phi: \mathcal{L}(X) \rightarrow \mathcal{L}(X)$ be a surjective continuous linear mapping. Then $r_{\phi(T)}(e)=r_{T}(e)$ for all $T \in \mathcal{L}(X)$ if and only if there exist an invertible operator $A \in \mathcal{L}(X)$ and $c \in \mathbb{C}$ of modulus 1 such that $A e=e$ and $\phi(T)=c A T A^{-1}$ for all $T \in \mathcal{L}(X)$.

Proof. Let $\phi(T)=c A T A^{-1}$ for all $T$. Then

$$
\begin{aligned}
r_{\phi(T)}(e) & =\limsup _{n \rightarrow \infty}\left\|(\phi(T))^{n} e\right\|^{1 / n}=\limsup _{n \rightarrow \infty}\left\|A T^{n} A^{-1} e\right\|^{1 / n} \\
& =\limsup _{n \rightarrow \infty}\left\|A T^{n} e\right\|^{1 / n}=\limsup _{n \rightarrow \infty}\left\|T^{n} e\right\|^{1 / n}=r_{T}(e) .
\end{aligned}
$$

Conversely, let $r_{\phi(T)}(e)=r_{T}(e)$ for all $T$. By Theorem $3.2, \phi$ is a spectral isometry and by Theorem 3.1, $\phi$ has one of the two forms mentioned there.

We will show that in the first case we can choose $A$ such that $A e=e$ and that the second case cannot occur.

(i) Let $\Phi(T)=c A T A^{-1}$ for all $T \in \mathcal{L}(X)$. Suppose first that the vectors $e$ and $A^{-1} e$ are linearly independent. Choose an operator $T \in \mathcal{L}(\mathcal{X})$ such that $T e=e$ and $T A^{-1} e=0$. Then $r_{T}(e)=1$ and

$$
r_{\phi(T)}(e)=\limsup _{n \rightarrow \infty}\left\|\phi(T)^{n} e\right\|^{1 / n}=\limsup _{n \rightarrow \infty}\left\|A T^{n} A^{-1} e\right\|^{1 / n}=0,
$$

a contradiction. Thus $A^{-1} e=\alpha e$ for some nonzero complex number $\alpha$. Set $A^{\prime}=\alpha A$. Then $\phi(T)=c A^{\prime} T A^{\prime-1}$ where $A^{\prime} e=\alpha A e=e$.

(ii) Let $\phi(T)=c A T^{*} A^{-1}$ for each $T \in \mathcal{L}(X)$, where $|c|=1$ and $A \in$ $\mathcal{L}\left(X^{*}, X\right)$ is invertible. Suppose that $\left\langle A^{-1} e, e\right\rangle=0$. Choose $T \in \mathcal{L}(X)$ such that $T e=e$ and $\operatorname{im}(T)=\mathbb{C} e$. Then $A^{-1} e \in \operatorname{im}(T)^{\perp}=\operatorname{ker}\left(T^{*}\right)$, and so $T^{*} A^{-1} e=0$. We have $r_{T}(e)=1$ and $r_{\phi(T)}(e)=\lim _{\sup _{n \rightarrow \infty}}\left\|A T^{* n} A^{-1} e\right\|^{1 / n}$ $=0$, a contradiction. Thus, $\left\langle A^{-1} e, e\right\rangle \neq 0$.

Choose a nonzero vector $x$ in $\operatorname{ker}\left(A^{-1} e\right)$ (such an $x$ exists if $\operatorname{dim} X \geq 2$; if $\operatorname{dim} X=1$, then the statement of the theorem is trivial). The vectors $e$ and $x$ are linearly independent. Let $T \in \mathcal{L}(X)$ be such that $T e=x, T x=x$, and $\operatorname{im}(T)=\mathbb{C} x$. Then $A^{-1} e \in \operatorname{im}(T)^{\perp}=\operatorname{ker}\left(T^{*}\right)$, and so $T^{*} A^{-1} e=0$. As in the previous case we have $r_{T}(e)=1$ and $r_{\phi(T)}(e)=0$, a contradiction. So, the second case cannot occur.

Problem 3.5. Is it possible to omit the assumption of continuity of $\phi$ in Theorems 3.3 and 3.4 ?

It is well known that every spectral isometry is automatically continuous. However, we used the continuity to prove that a surjection preserving the local spectrum (local spectral radius) is a spectral isometry.

Acknowledgements. The paper was written when the first author had a visiting position at the Mathematical Institute of the Czech Academy of Sciences. He wishes to express his thanks for the hospitality there. 
The research was partially supported by a joint Czech-Slovenian grant BI-CZ/07-08-013. The second author was also supported by grant No. 201/09/0473 of GA ČR.

\section{References}

[1] A. Bourhim and V. G. Miller, Linear maps on $M_{n}(\mathbb{C})$ preserving the local spectral radius, Studia Math. 188 (2008), 67-75.

[2] A. Bourhim and T. Ransford, Additive maps preserving the local spectrum, Integral Equations Operator Theory 55 (2006), 377-385.

[3] M. Brešar and P. Šemrl, Linear maps preserving the spectral radius, J. Funct. Anal. 142 (1996), 360-368.

[4] J. Daneš, On local spectral radius, Časopis Pěst. Mat. 112 (1987), 177-187.

[5] M. González and M. Mbekhta, Linear maps on $M_{n}(\mathbb{C})$ preserving the local spectrum, Linear Algebra Appl. 427 (2007), 176-182.

[6] K. B. Laursen and M. M. Neumann, An Introduction to Local Spectral Theory, London Math. Soc. Monogr. 20, Clarendon Press, Oxford, 2000.

[7] V. Müller, Spectral Theory of Linear Operators and Spectral Systems in Banach Algebras, 2nd ed., Oper. Theory Adv. Appl. 139, Birkhäuser, Basel, 2007.

[8] P. Vrbová, On local spectral properties of operators in Banach spaces, Czechoslovak Math. J. 23 (1973), 483-492.

IMFM, University of Ljubljana

Jadranska ul. 19

SI-1000 Ljubljana, Slovenia

E-mail: janko.bracic@fmf.uni-lj.si
Mathematical Institute Czech Academy of Sciences

Žitná 25

11567 Praha 1, Czech Republic E-mail: muller@math.cas.cz

Received October 22, 2008

Revised version March 27, 2009 